\title{
EMIGRAÇÃO INTERNACIONAL E TROCAS POPULACIONAIS INTERNAS DE MINAS GERAIS, DE VALADARES E DE IPATINGA, NO DECÊNIO 1981/1991
}

Weber Soares ${ }^{1}$

\section{1- INTRODUÇÃO}

A trajetória analítica empreendida neste trabalho tem o objetivo de discriminar a contribuição das trocas populacionais internas e internacionais à dinâmica migratória da microrregião de Governador Valadares, da microrregião de Ipatinga e de Minas Gerais. Para tanto, o primeiro esforço consistirá em descrever as técnicas de mensuração dos fluxos populacionais, tanto a direta quanto a indireta: aquela técnica lida com dados resultantes de resposta a quesitos diretamente ligados à migração, e esta consiste no tratamento de dados indiretamente ligados à migração. Se forem adotados certos pressupostos e hipóteses, a técnica indireta permite estimar, geralmente por resíduo, os efeitos líquidos (saldos migratórios), dos deslocamentos populacionais (CARVALHO, 1980).

Vale notar que a estimação dos emigrantes internacionais referentes ao quinquênio 1986/1991 para os recortes territoriais em pauta toma como referência a linha de procedimentos metodológicos desenvolvida por CARVALHO \& RIGOTTI (1999), RIGOTTI (1999), CARVALHO et al (2000a, b, c) e CARVALHO et al (2001).

Por fim, à luz de tais técnicas e dos dados censitários de 1970, de 1980 e de 1991 serão estimados, tanto para as duas microrregiões mineiras, a valadarense e a ipatinguense, quanto para o estado de Minas Gerais, o saldo migratório decenal (1981/1991), os saldos migratórios quinquenais, o de 1981/1986 e o de 1986/1991, e o número de emigrantes internacionais do quinquênio 1986/1991.

\footnotetext{
${ }^{1}$ Professor e Pesquisador do Departamento de Geografia da UFMG

Cadernos do Leste

Artigos Científicos

Belo Horizonte, Edição Especial, 2000 a 2008
} 


\section{2- TÉCNICAS DE MENSURAÇÃO DOS FLUXOS POPULACIONAIS}

Crescente atenção tem sido dada aos fluxos migratórios nos censos brasileiros. As questões sobre migração, no Censo de 1970, foram direcionadas apenas aos não-naturais dos municípios. Os quesitos referiam-se ao tempo de residência, sem interrupção, na Unidade da Federação (UF) e no município, ao lugar de procedência (UF ou país estrangeiro) e à situação do domicílio (urbano ou rural). A natureza e as possíveis combinações das respostas a tais quesitos não permitem mensurar a migração dos naturais do município ou da UF (migração de retorno), que pode, em certas condições, alcançar valores significativos (CARVALHO, 1980).

Além de ter mantido, basicamente, os mesmos quesitos sobre os fluxos populacionais internos do Censo de 1970, o Censo de 1980 trouxe duas grandes novidades: i) a investigação não se limitou aos não-naturais e, além das migrações intermunicipais, foi pesquisada a migração intramunicipal (entre os setores rural e urbano); e ii) foi identificado o nome do município de residência anterior das pessoas com menos de dez anos de residência no município atual (na data do censo). Com isso, o cálculo dos ganhos (imigrantes) e das perdas (emigrantes) de população, entre duas unidades espaciais analisadas, tornou-se factível; todavia, a diferença entre imigrantes e emigrantes de tais unidades representa algo próximo ao conceito de saldo migratório, mas não o ganho líquido de população oriundo do processo migratório entre duas datas fixas. As lacunas relacionadas à migração rural-urbana dentro do próprio município bem como à migração de retorno foram suprimidas (CARVALHO, 1985).

O Censo de 1991 é o mais completo no tocante aos dados sobre migração, pois além de manter os quesitos do censo anterior trouxe uma inovação: a informação de "data fixa", obtida por meio do quesito 21 (Indique a sigla da UF e o nome do município ou país em que residia em 1/9/86.), o que amplia as possibilidades de pesquisa nesse campo. Imigrantes e emigrantes identificados com base nesse quesito guardam semelhança conceitual com os 
imigrantes e emigrantes implícitos no saldo migratório resultante de técnica indireta (CARVALHO \& MACHADO, 1992).

\section{Mensuração indireta da migração}

A técnica de estimação do saldo migratório (SM) consiste, no final de certo período, na diferença entre a população observada e a população esperada (fechada): corresponde ao resultado líquido dos movimentos populacionais. Não inclui as pessoas que, dentro do período, saíram e retornaram nem as que entraram e saíram. As estimativas obtidas por esse tipo de técnica correspondem apenas aos saldos migratórios, sem desagregação de imigrantes e emigrantes.

Dados dois censos consecutivos e um intervalo de " $n$ " anos entre eles, deve ser considerado imigrante de data fixa, todo o residente na unidade espacial pesquisada, com idade superior a " $n$ " anos no segundo censo, que, por ocasião do primeiro censo, residia em outra unidade; e, emigrante de data fixa, de determinada unidade, toda a pessoa, com mais de " $\mathrm{n}$ " anos no segundo censo, recenseada no primeiro censo e residente em outro lugar no segundo censo.

Os nascidos no período intercensitário, que sobrevivem até a data do último censo, filhos de pais migrantes, respondem pelos efeitos diretos e indiretos da migração, que têm de ser levados em conta no saldo migratório. Os efeitos diretos correspondem à diferença entre as crianças, sobreviventes no final do período, que entraram (imigrantes) em determinado lugar, e aí permaneceram, e as crianças que saíram (emigrantes) desse lugar, e a ele não retornaram até a data do segundo censo. Já os efeitos indiretos correspondem à diferença entre os filhos sobreviventes de imigrantes do período, que nasceram na região de destino, no período considerado, não morreram e dela não saíram e os filhos de emigrantes que nasceram no lugar de destino, não morreram e não retornaram à região de origem (CARVALHO, 1980).

Cadernos do Leste

Artigos Cientificos

Belo Horizonte, Edição Especial, 2000 a 2008 
A diferença entre população esperada e população observada corresponde ao verdadeiro conceito de saldo migratório que é a contribuição líquida, entre duas datas fixas, dos fluxos migratórios ao tamanho da população. Consequentemente, o saldo migratório é o resultado de dois componentes: os imigrantes de data fixa (positivo) e os emigrantes de data fixa (negativo).

Ainda no campo das mensurações indiretas, a taxa líquida de migração constitui estimativa importante para a análise das migrações e pode ser construída de duas formas: pela razão entre o saldo migratório e a população esperada, fechada, no final do período ou pela razão entre o saldo migratório e a população observada, também no final do período. Neste caso, a taxa líquida de migração representa a “... proporção da população observada no segundo censo resultante do processo migratório, quando a taxa for positiva, e a proporção em que a população seria acrescida na ausência de migração, se negativa" (CARVALHO \& RIGOTTI, 1999). Naquele caso, a taxa líquida de migração informa a redução proporcional da população fechada, se negativa, ou ganho proporcional, se positiva, devido ao fluxo migratório do período.

\section{Mensuração direta da migração}

A mensuração direta serve-se dos quesitos censitários para identificar os migrantes de última etapa ou de data fixa. O conceito de última etapa não se confunde com a informação relativa ao "lugar de última residência", porque esta informação não traz a referência temporal do fluxo migratório. É da combinação das respostas aos quesitos censitários "tempo de residência" e "lugar de última residência", dadas pelas pessoas que moram há menos de 10 anos em certo local, que a última etapa da trajetória dos migrantes do decênio, contemplando tanto a dimensão temporal, quanto espacial do deslocamento, torna-se conhecida.

Desse modo, os dados dos dois quesitos sobre a última etapa revelam os movimentos populacionais diretos entre duas unidades espaciais; expõem, pela diferença entre os imigrantes que residem há menos de 10 anos num lugar e os emigrantes desse mesmo lugar que moram fora há menos de 10 anos, algo próximo ao conceito de saldo migratório.

Cadernos do Leste

Artigos Cientificos

Belo Horizonte, Edição Especial, 2000 a 2008 
A diferença entre imigrantes e emigrantes de datas fixas faz saber, por sua vez, o saldo migratório entre cada par de unidades geográficas ao final do período, ou seja, acusa a contribuição das migrações ao crescimento populacional (CARVALHO \& RIGOTTI, 1999). O saldo migratório obtido com base no quesito de data fixa apresenta as seguintes vantagens, com relação às estimativas por técnica indireta: i) resulta de mensuração direta e não de estimativas; ii) pode ser calculado para pares de unidades geográficas, isto é, não se limita às trocas populacionais entre determinada unidade geográfica e o resto do mundo; e iii) pode ser decomposto de acordo com o número de imigrantes e emigrantes. Além disso, a análise da migração de retorno pleno, constituída das pessoas que saíram do lugar de residência e a esse mesmo lugar retornaram entre duas datas fixas, é exeqüível nesse caso (CARVALHO \& MACHADO, 1992).

\section{Saldo migratório e taxas líquidas de migração decenais: estimação}

O saldo migratório intercensitário é, por meio de técnica indireta, estimado por resíduo, isto é, pela diferença entre a população observada e a esperada (fechada) no final do período. Essa diferença, para as pessoas que têm idade superior ao intervalo intercensitário, corresponde aos efeitos diretos da migração; para os que têm idade inferior, aos efeitos diretos e indiretos (CARVALHO \& RIGOTTI, 1999).

Para obter a população esperada de certa região, no segundo censo, é preciso multiplicar a população aí observada, no primeiro censo, pela relação de sobrevivência representativa do período em questão. Assim:

$$
{ }_{\boldsymbol{m}} \boldsymbol{P}_{\boldsymbol{x}+\boldsymbol{n}, \boldsymbol{e s p} \boldsymbol{p}}^{\boldsymbol{j} \boldsymbol{n}}{ }_{\boldsymbol{m}} \boldsymbol{P}_{\boldsymbol{x}, \boldsymbol{o b}}^{\boldsymbol{j}, 0} \times{ }_{\boldsymbol{m}}^{\boldsymbol{n}} \boldsymbol{S}_{\boldsymbol{x}}^{\boldsymbol{j}} \quad \text { (EQUAÇ̃̃̃ 1) }
$$

em que ${ }_{\boldsymbol{m}} \boldsymbol{P}_{\boldsymbol{x}+\boldsymbol{n}, \boldsymbol{e s p}}^{\boldsymbol{j , \boldsymbol { n }}}$ é a população esperada na região j, do grupo etário $\mathrm{x}+\mathrm{n}, \mathrm{x}+\mathrm{n}+\mathrm{m}$, no segundo censo (ano n); ${ }_{\boldsymbol{m}} \boldsymbol{P}_{\boldsymbol{x}, \boldsymbol{o b}}^{\boldsymbol{j}, 0}$ é população observada na região j, no primeiro censo (ano 0), 
do grupo etário $\mathrm{x}, \mathrm{x}+\mathrm{m}$; e ${ }_{\boldsymbol{m}}^{\boldsymbol{n}} \boldsymbol{S}_{\boldsymbol{x}}^{j}$ é a relação de sobrevivência do grupo etário $\mathrm{x}, \mathrm{x}+\mathrm{m}$, durante o período de $\mathrm{n}$ anos (do ano 0 ao ano $\mathrm{n}$ ).

Com esse resultado, calcula-se o saldo migratório decenal, por meio da seguinte expressão:

$$
{ }_{\boldsymbol{m}}^{n} \boldsymbol{S} \boldsymbol{M}_{\boldsymbol{x}+\boldsymbol{n}}^{\boldsymbol{j , n}}={ }_{\boldsymbol{m}} \boldsymbol{P}_{\boldsymbol{x}+\boldsymbol{n}, \boldsymbol{o b}}^{\boldsymbol{j}, \boldsymbol{n}}-{ }_{\boldsymbol{m}} \boldsymbol{P}_{\boldsymbol{x}+\boldsymbol{n}, \boldsymbol{e s p}}^{\boldsymbol{j}, \boldsymbol{n}} \quad \text { (EQUAÇ̃̃O 2), }
$$

na qual ${ }_{\boldsymbol{m}}^{\boldsymbol{n}} \boldsymbol{S} \boldsymbol{M}_{\boldsymbol{x}+\boldsymbol{n}}^{\boldsymbol{j}, \boldsymbol{n}}$ corresponde ao saldo migratório de $\mathrm{n}$ anos.

A divisão do saldo migratório pela população observada ou pela população esperada (fechada), no segundo censo, leva ao conhecimento da taxa líquida de migração. Então:

$$
\begin{aligned}
& { }_{m}^{n} T L M_{x+n}^{j, n}=\frac{{ }_{m}^{n} S M_{x+n}^{j, n}}{{ }_{m} P_{x+n, o b}^{j, n}} \quad \text { (EQUAÇ̃̃) 3) } \\
& \text { ou } \\
& { }_{m}^{n} T L M_{x+n}^{j, n}=\frac{{ }_{m}^{n} S M_{x+n}^{j, n}}{{ }_{m} P_{x+n, e s p}^{j, n}} \quad \text { (EQUAÇÃO 4). }
\end{aligned}
$$

Em ambas as equações, ${ }_{\boldsymbol{m}}^{\boldsymbol{n}} \boldsymbol{T} \boldsymbol{L} \boldsymbol{M}_{\boldsymbol{x}+\boldsymbol{n}}^{\boldsymbol{j , n}}$ representa a taxa líquida de migração, que varia de acordo com o denominador escolhido.

Para estimar a população esperada, CARVALHO \& RIGOTTI (1999) recomendam, devido aos erros de declaração de idade e à deficiência de cobertura censitária, o uso das razões intercensitárias de sobrevivência do país em vez de relações de sobrevivência retiradas de tabelas de mortalidade da região. Visto que o recurso a esse método implica o pressuposto de que, no período, a população do país tenha se mantido fechada, o que não ocorreu no 
Brasil, na década de $80^{2}$, uma maneira de ajustar a razão intercensitária de sobrevivência de uma região qualquer, para os anos 80 , com base na razão intercensitária de sobrevivência do país, nos anos $70^{3}$ e no quociente entre as relações de sobrevivência da região na década de 80 e do país no decênio anterior, é proposta por CARVALHO \& RIGOTTI (1999). Essas relações de sobrevivência, referentes às décadas de 1970 e de 1980, procedem de tabelas de sobrevivência construídas, respectivamente, para a região e para o país. Logo, a estimativa da razão intercensitária de sobrevivência regional ganha expressão formal na seguinte equação:

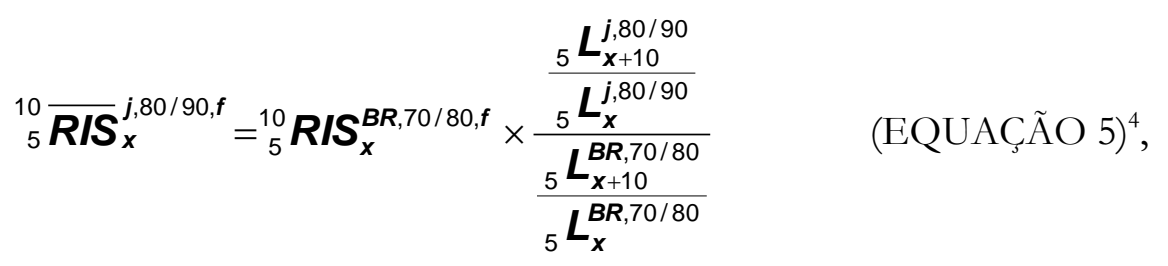

na qual ${ }_{5}^{10} \overline{\boldsymbol{R I S}}_{\boldsymbol{x}}^{\boldsymbol{j}, 80 / 90, \boldsymbol{f}}$ é, para os anos 80, a razão intercensitária de sobrevivência da população estimada fechada da região j, para as idades $\mathrm{x}, \mathrm{x}+5 ;{ }_{5}^{10} \boldsymbol{R} \boldsymbol{S}_{\boldsymbol{x}}^{\boldsymbol{B R}, 70 / 80, \boldsymbol{f}}$ simboliza a razão intercensitária de sobrevivência observada do Brasil para as idades $\mathrm{x}, \mathrm{x}+5$, nos anos 70 ; e ${ }_{5} L_{\boldsymbol{x}}^{\boldsymbol{j}, 80 / 90},{ }_{5} \boldsymbol{L}_{\boldsymbol{x}+10}^{\boldsymbol{j}, 80 / 90},{ }_{5} \boldsymbol{L}_{\boldsymbol{x}}^{\boldsymbol{B R}, 70 / 80}$ e ${ }_{5} \boldsymbol{L}_{\boldsymbol{x}+10}^{\boldsymbol{B R}, 70 / 80}$ correspondem, para os grupos etários $\mathrm{x}, \mathrm{x}+5$ e $x+10, x+15$, às relações de sobrevivência retiradas das tabelas de mortalidade da região j, relativa à década de 80 , e do Brasil, correspondente aos anos 70.

Em relação aos grupos etários com idade igual ou superior a 15 e inferior a 65 anos, a população esperada, em 1990, de determinada região é, então, o produto da população aí observada em 1980 e das relações intercensitárias de sobrevivência ajustadas para essa mesma região, no período 1980/1990. É o que põe em evidência a equação seguinte:

\footnotetext{
${ }^{2}$ A emigração internacional foi significativa nesse período, atestam as estimativas feitas por SOARES (1995), CARVALHO (1996), GARCIA \& SOARES (1999), RIGOTTI (1999), CARVALHO et al (2000a), CARVALHO et al (2000b), CARVALHO et al (2000c) e CARVALHO et al (2001) para certas regiões, para alguns estados e para todo o Brasil.

${ }^{3}$ A população do Brasil pode ser considerada, nessa década, fechada.

${ }^{4}$ O desenvolvimento algébrico dessa expressão matemática acha-se em CARVALHO \& RIGOTTI (1999).

Cadernos do Leste

Artigos Cientificos

Belo Horizonte, Edição Especial, 2000 a 2008
} 


$$
{ }_{\boldsymbol{m}} \boldsymbol{P}_{\boldsymbol{x}+\boldsymbol{n}, \boldsymbol{e s p} \boldsymbol{p}}^{\boldsymbol{j}, 90}{ }_{\boldsymbol{m}} \boldsymbol{P}_{\boldsymbol{x}, \boldsymbol{o b}}^{\boldsymbol{j}, 80} \times{ }_{5}^{10} \overline{\boldsymbol{R I S}}_{\boldsymbol{x}}^{\boldsymbol{j}, 80 / 90, \boldsymbol{f}} \quad \text { (EQUAÇÃO 6) }
$$

A diferença, em 1990, entre a população observada e a população esperada da região j, estimada por meio do procedimento descrito acima, constitui o saldo migratório decenal dos que tinham entre 15 e 64 anos de idade: estarão incluídas nessas estimativas as trocas populacionais que, porventura, tenham ocorrido entre a região e outros países (CARVALHO \& RIGOTTI, 1999). Porém, quando se leva em conta a migração internacional, sobretudo que a recentidade desse fenômeno sugere participação nula ou muito pequena dos mais jovens e dos mais velhos, seria razoável considerar os grupos etários de 10 a $14^{5}$, de 65 a 69 e de 70 anos e mais fechados a fluxos dessa natureza, na década de 80.

Para tanto, a correção das razões intercensitárias de sobrevivência da população fechada regional, referentes aos grupos etários de 10 a 14, 65 a 69 e 70 anos e mais foi feita por intermédio das razões intercensitárias de sobrevivência do país, na década de $80^{6}$, em vez de recorrer às razões intercensitárias da década de 70, e das relações de sobrevivência $\left(\mathrm{L}_{\mathrm{x}}\right)$ oriundas das tabelas de mortalidade da região e do país, no mesmo período. Portanto:

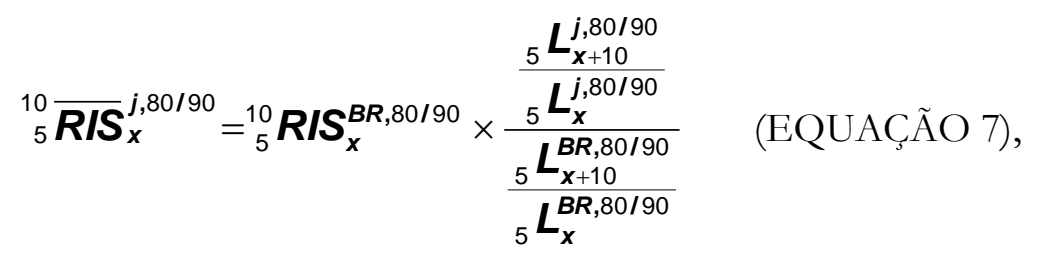

na qual ${ }_{5}^{10} \boldsymbol{R} \boldsymbol{S}_{\boldsymbol{x}}^{\boldsymbol{B R}, 80 / 90}$ representa a razão intercensitária de sobrevivência observada do Brasil para as idades $\mathrm{x}, \mathrm{x}+5$, nos anos 80; e ${ }_{5} \boldsymbol{L}_{\boldsymbol{x}}^{\boldsymbol{B R}, 70 / 80}$ e ${ }_{5} \boldsymbol{L}_{\boldsymbol{x}+10}^{\boldsymbol{B R}, 70 / 80}$ referem-se às relações de

\footnotetext{
${ }^{5}$ Nas estimativas realizadas para a microrregião de Valadares, pareceu razoável considerar, mediante pesquisa que dá conta do caráter mais antigo e de certas medidas sobre a migração internacional da sede do município de Governador Valadares (SOARES, 1995), o grupo etário de 10 a 14 anos aberto a esse tipo de migração, nos anos 80 .

${ }^{6}$ A taxa média anual de crescimento geométrico do período 1980/1991, por grupo etário, facultou o ajuste da população brasileira residente em 1991 para 1990.
}

Cadernos do Leste

Artigos Científicos

Belo Horizonte, Edição Especial, 2000 a 2008 
sobrevivência dos grupos etários $\mathrm{x}, \mathrm{x}+5$ e $\mathrm{x}+10, \mathrm{x}+15$, retiradas da tabela de mortalidade do Brasil relativa à década de 80 .

Não deve restar dúvida sobre a necessidade de que as funções de mortalidade utilizadas, a qualidade dos dados básicos e os pressupostos desse método devem ser objeto de cuidadoso exame, em consequência dos vieses que cada um desses componentes pode provocar nas estimativas dos saldos migratórios realizadas por técnica indireta.

Quanto ao saldo migratório decenal das pessoas pertencentes aos dois primeiros grupos etários ( 0 a 4 anos e 5 a 9 anos), o recurso à Razão Criança Mulher, proposta por LEE (KUZNETS \& THOMAS (1957), faculta tal estimativa. O produto da razão entre o número de crianças de 0 a 4 anos e o número de mulheres de 15 a 44 anos, na população observada, no final do período intercensitário, pelo saldo migratório das mulheres de 15 a 44 anos traz à luz o saldo migratório dessas crianças.

\section{Saldo migratório e taxas líquidas do primeiro quinquenais e emigrantes internacionais do segundo quinquênio: estimação}

Saldos migratórios, nos anos 80, de estados, de regiões e de municípios, dependendo do tamanho da população, podem ser calculados para o primeiro quinquênio (1980/1985 ou 1981/1986 - neste caso, com o ajuste da população residente para 1981), e para o segundo quinquênio (1985/1990 ou 1986/1991 - naquele caso, com o ajuste da população para 1985) (CARVALHO \& MACHADO, 1992).

A associação de técnicas de mensuração direta com técnicas de estimação indireta faculta estimativas dos saldos migratórios internacionais e do número de emigrantes internacionais do período coberto pela informação de data fixa, para certas unidades geográficas.

\section{Saldo migratório e taxas líquidas do primeiro quinquênio}


Para o primeiro quinquênio (1981/1986), a estimativa do saldo migratório da região j, dos que tinham cinco anos ou mais em 1986, só pode ser obtida por técnica indireta, o que implica a estimação tanto da população residente, quanto da população esperada, no final do recorte temporal.

Como, no meio da década de 80 , não foi feita nenhuma contagem populacional no país, um dos recursos para estimar a população residente da região j, nesse marco temporal, consiste na interpolação: a taxa geométrica média anual de crescimento, calculada para o período 1981/1991, por coorte de nascimento, facultaria tal estimativa. Essa forma de interpolação supõe que o crescimento, ao longo do período, tenha se dado a uma taxa constante (consequência do efeito combinado da mortalidade e da migração), o que não ocorre necessariamente: algumas coortes da população residente estimada para o meio do período podem estar subestimadas, e outras, sobre estimadas ${ }^{6}$ (CARVALHO et al., 2000b).

O método "Relações de Coortes", proposto por DUCHESNE (1989) e utilizado aqui para estimar a população residente da região j, em 1986, lança mão das razões de sobrevivência decenais das coortes da população aberta - na sobrevivência estão incluídos os efeitos da migração -, para a estimação de razões de sobrevivência quinquenais dessa mesma população da região j. Esse procedimento reduz os problemas relacionados com o pressuposto de que o crescimento/decrescimento do número de pessoas na coorte, durante o período entre o primeiro e segundo censo, tenha se dado a uma taxa constante (CARVALHO et al, 2000b).

O próximo passo, para encontrar o saldo migratório da região j, no primeiro quinquênio do período 1981/1991, consiste em estimar a população esperada (fechada) para o mesmo ano, isto é, 1986.

As estimativas das razões de sobrevivência da população fechada - nesse caso, não estão incluídos os efeitos das migrações -, foram obtidas por meio do mesmo procedimento adotado para estimar as razões de sobrevivência quinquenais da população aberta, proposto por Duchesne.

Cadernos do Leste

Artigos Científicos

Belo Horizonte, Edição Especial, 2000 a 2008 
A razão de sobrevivência do primeiro quinquênio, que deve ser usada para estimar a população esperada (fechada), no meio da década de 80, é dada, então, pela média que toma os valores constantes na equação seguinte:

$$
{ }_{5}^{5} \overline{\boldsymbol{R S}}_{\boldsymbol{x}+5}^{j, I}=\frac{\sqrt{{ }_{5}^{10} \overline{\boldsymbol{R I S}}_{\boldsymbol{x}}^{j}}+\sqrt{{ }_{5}^{10} \overline{\boldsymbol{R I S}}_{\boldsymbol{x}+5}^{j}}}{2} \quad \text { (EQUAÇÃO 8) }
$$

na qual ${ }_{5}^{5} \overline{\boldsymbol{R S}}_{\boldsymbol{x}+5}^{j, \boldsymbol{I}}$ é a razão de sobrevivência quinquenal, da população fechada, estimada para a região j, relativa ao primeiro quinquênio, do grupo etário $\mathrm{x}+5, \mathrm{x}+10 ;{ }_{5}^{10} \overline{\boldsymbol{R I S}}_{\boldsymbol{x}}^{\boldsymbol{j}, \boldsymbol{f}}$ refere-se à razão intercensitária de sobrevivência decenal da população fechada, estimada para a região j, do grupo etário x, x+5; e ${ }_{5}^{10} \overline{\boldsymbol{R I S}}_{\boldsymbol{x}+5}^{j}$ simboliza a razão intercensitária de sobrevivência decenal estimada, do grupo etário $x+5, x+10$, da população fechada da região $j$.

Em relação ao quinquênio 1986/1991, as razões de sobrevivência da população fechada, indispensáveis, como será visto na sequência, para estimar o saldo migratório desse período, resultam da equação que se segue:

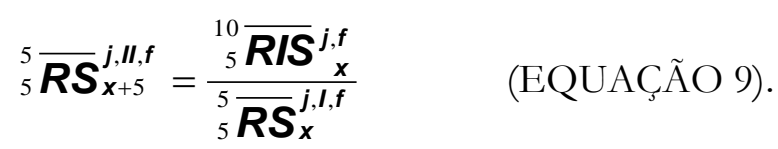

A população esperada da região j, em 1986, para os grupos etários de 5 anos e mais, é o produto da população aí observada, em 1981, pela razão de sobrevivência da população fechada, estimada do primeiro quinquênio. É o que acusa a equação:

$$
\left.{ }_{5} \boldsymbol{P}_{\boldsymbol{x}+5, \boldsymbol{e s p} \boldsymbol{j}}^{\boldsymbol{j}, 86} \boldsymbol{P}_{\boldsymbol{x}, \boldsymbol{o b}}^{\boldsymbol{j}, 81} \times{ }_{5}^{5} \overline{\boldsymbol{R S}}_{\boldsymbol{x}}^{\boldsymbol{j , \boldsymbol { I }}} \quad \text { (EQUAÇ } \tilde{\mathrm{AO}} 100\right)
$$

em que ${ }_{5} \boldsymbol{P}_{\boldsymbol{x}+5, \text { esp }}^{j, 86}$ é a população esperada (fechada) de idade $\mathrm{x}+5, \mathrm{x}+10$, em 1986, da região j; e ${ }_{5} \boldsymbol{P}_{\boldsymbol{x}, \boldsymbol{o b}}^{\boldsymbol{j}, 81}$ exprime a população de x, x+5 anos observada na região j, em 1981. 
Estimadas as populações observada e esperada para o meio da década, o saldo migratório consiste em subtrair esta população daquela, como faz ver a fórmula:

$$
{ }_{5}^{5} \boldsymbol{S} \boldsymbol{M}_{\boldsymbol{x}+5}^{\boldsymbol{j} \boldsymbol{I}}={ }_{5} \boldsymbol{P}_{\boldsymbol{x}+5, \text { est }}^{\boldsymbol{j , 8 6}}-{ }_{5} \boldsymbol{P}_{\boldsymbol{x}+5, \text { esp }}^{\boldsymbol{j}, 86} \quad \text { (EQUAÇÃO 11) }
$$

na qual ${ }_{5}^{5} \boldsymbol{S M}_{\boldsymbol{x}+5}^{\boldsymbol{j}, \boldsymbol{I}}$ é o saldo migratório da região j, referente ao período 1981/1986. No tocante ao saldo migratório das crianças de 0 a 4 anos, o procedimento de estimação consiste em multiplicar o saldo migratório da população feminina de 15 a 44 anos, do quinquênio 1981/1986, pela média das razões criança-mulher referentes ao primeiro e ao segundo censos.

A relação entre o saldo migratório do quinquênio $1981 / 1986\left({ }_{5}^{5} \boldsymbol{S} \boldsymbol{M}_{\boldsymbol{x}+5}^{\boldsymbol{j}, \boldsymbol{I}}\right)$ e a população residente estimada $\left({ }_{5} \boldsymbol{P}_{\boldsymbol{x}+5, \text { est }}^{j, 86}\right)$ para 1986 constitui a taxa líquida de migração $\left({ }_{5}^{5} \boldsymbol{T L} \boldsymbol{M}_{\boldsymbol{x}+5}^{\boldsymbol{j , l}}\right)$, atinente ao mesmo período (1981/1986). Então:

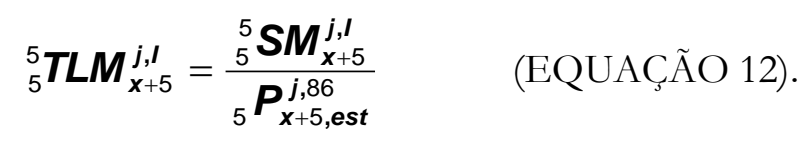

\section{Saldo migratório e taxas líquidas de migração do segundo quinquênio}

Para as pessoas com idade igual ou superior a 10 anos em 1991, o saldo migratório do quinquênio 1986/1991, na região j, decorre da diferença entre os que aí residiam em 1991 e a população esperada fechada, ao final do quinquênio 1986/1991. Como mostra a equação que se segue, essa população esperada equivale ao produto da razão intercensitária de sobrevivência do segundo quinquênio pela população residente estimada em 1986. Então,

$$
{ }_{5} \boldsymbol{P}_{\boldsymbol{x}+10, \mathbf{e s p}}^{\boldsymbol{j}, 91}={ }_{5} \boldsymbol{P}_{\boldsymbol{x}+5, \boldsymbol{e s t}}^{\boldsymbol{j}, 86} \times{ }_{5}^{5} \overline{\boldsymbol{R S}}_{\boldsymbol{x}+5}^{\boldsymbol{j}, \boldsymbol{I I}} \quad \text { (EQUAÇÃO 13) }
$$

Cadernos do Leste 
Nessa expressão matemática, ${ }_{5} \boldsymbol{P}_{\boldsymbol{x}+10, \mathbf{e s p}}^{j, 91}$ denota a população do grupo etário $\mathrm{x}+5$, x+10 anos esperada (fechada) na região j, em 1991. CARVALHO et al (2000c) observam que a população esperada fechada no final do segundo quinquênio, em 1991, é diferente da esperada fechada no final do decênio, também em 1991, porque, no primeiro caso, o período considerado é de 5 anos (fechada entre 1986 e 1991) e, no último, de 10 anos (fechada entre 1981 e 1991).

Pode, enfim, o saldo migratório do período 1986/1991, com a estimativa da população esperada na região j, ser calculado pela seguinte operação matemática:

$$
{ }_{5}^{5} \boldsymbol{S} \boldsymbol{M}_{\boldsymbol{x}+10}^{\boldsymbol{j}, \boldsymbol{l l}}={ }_{5} \boldsymbol{P}_{\boldsymbol{x}+10, \mathbf{o b}}^{\boldsymbol{j}, 91}-{ }_{5} \boldsymbol{P}_{\boldsymbol{x}+10, \mathbf{e s p}}^{\boldsymbol{j}, 86} \quad \text { (EQUAÇÃO 11) }
$$

na qual ${ }_{5}^{5} \mathbf{S M}_{\boldsymbol{x}+10}^{\boldsymbol{j}, \text { II }}$ é o saldo migratório do quinquênio 1986/1991, para os que tinham 10 anos ou mais de idade em 1991, na região j.

Vale ressaltar que o saldo migratório dos grupos etários de 0 a 4 e de 5 a 9 anos resulta da multiplicação da razão criança-mulher, observada em 1991, pelo saldo migratório da população feminina em idade reprodutiva: a razão, cujo numerador são as crianças de 0 a 4 anos, do sexo masculino ou feminino, conforme o caso, tem como denominador a população feminina de 15 a 44 anos; o denominador são as mulheres de 20 a 49 anos, quando a razão criança-mulher tem as crianças de 5 a 9 anos como numerador.

A relação entre o saldo migratório do quinquênio $1986 / 1991\left({ }_{5}^{5} \mathbf{S} \boldsymbol{M}_{\boldsymbol{x}+10}^{\boldsymbol{j}, \boldsymbol{l l}}\right)$ e a população observada $\left({ }_{5} \boldsymbol{P}_{\boldsymbol{x}+10, \boldsymbol{o b}}^{j, 91}\right)$ em 1991 dá origem à taxa líquida de migração $\left({ }_{5}^{5} \boldsymbol{T} L \boldsymbol{M}_{\boldsymbol{x}+10}^{\boldsymbol{j}, \boldsymbol{I I}}\right)$, referente ao período (1986/1991). Logo:

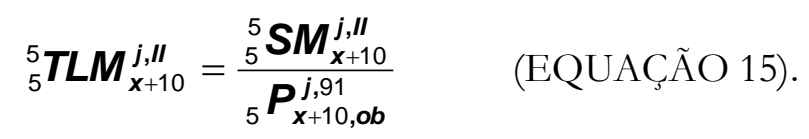

Não é certo considerar o saldo migratório decenal como simples soma dos saldos quinquenais, pois as pessoas que fazem parte do saldo do primeiro quinquênio estão expostas, por um período de mais cinco anos, até o final do decênio, ao risco de morrer. $O$ saldo

Cadernos do Leste

Artigos Cientificos

Belo Horizonte, Edição Especial, 2000 a 2008 
decenal é, isto sim, a soma do saldo dos últimos cinco anos mais os sobreviventes do saldo migratório do primeiro quinquênio (CARVALHO \& RIGOTTI, 1999), como registra a expressão:

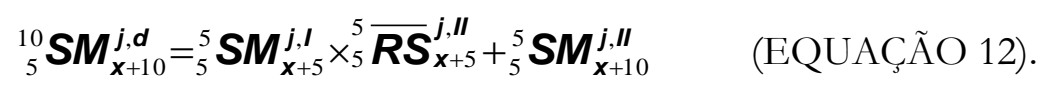

Operações algébricas registram que a taxa líquida de migração decenal não corresponde ao produto das taxas líquidas de migração quinquenais. Quando as taxas líquidas têm como denominador a população observada no final do período, a taxa líquida de migração decenal é a soma das duas taxas líquidas de migração quinquenais do período menos o produto delas (CARVALHO \& RIGOTTI, 1999). Então:

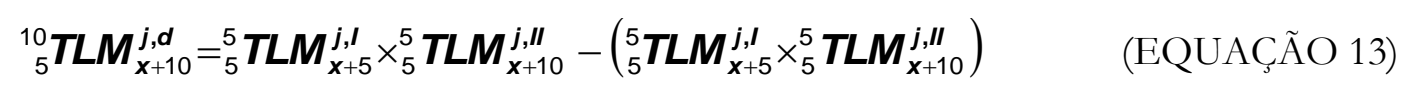

\section{Emigrantes internacionais do quinquênio 1986/1991}

Para estimar os emigrantes internacionais do período 1986/1991, o procedimento inicial consiste em extrair do quesito referente ao lugar de residência em data fixa passada, no Censo Brasileiro de 1991, o número de imigrantes intra e internacionais, bem como o número de emigrantes intranacionais da região j. Em seguida, a diferença entre o total de imigrantes (intra e internacionais) e o saldo migratório, estimado por meio de técnica indireta (seção anterior) para o segundo quinquênio, leva à estimativa do total de emigrantes (intra e internacionais). Desse total de emigrantes, deve ser excluída a participação dos emigrantes intranacionais de data fixa da região, para encontrar o número aproximado de emigrantes internacionais do segundo quinquênio dos anos 80. Formalmente, isso pode ser expresso da seguinte maneira:

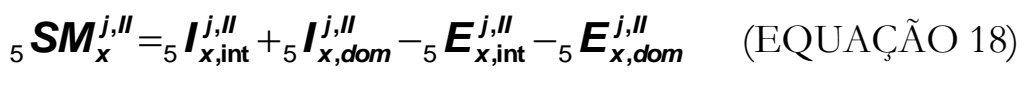

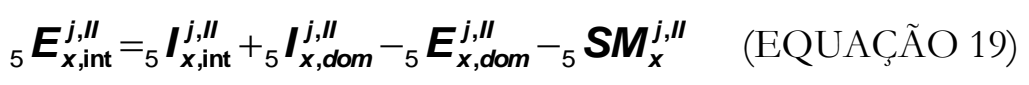

Cadernos do Leste

Artigos Cientificos 
${ }_{5} \boldsymbol{S M}_{\boldsymbol{x}}^{\boldsymbol{j}, \boldsymbol{I I}}$ é, para o período 1986/1991, o saldo migratório da região j; ${ }_{5} \boldsymbol{E}_{\boldsymbol{x}, \text { int }}^{\boldsymbol{j}, \boldsymbol{I I}}$ corresponde aos emigrantes internacionais da região j, para o mesmo período; ${ }_{5} \boldsymbol{I}_{\boldsymbol{x}, \text { int }}^{j, \boldsymbol{I I}}$ refere-se ao número de imigrantes internacionais de data fixa, do quinquênio 1986/1991; ${ }_{5} \boldsymbol{I}_{\boldsymbol{x}, \boldsymbol{d o m}}^{\boldsymbol{j} \text {,II }}$ representa os imigrantes de data fixa, do mesmo quinquênio, cuja origem foram outras regiões brasileiras; e ${ }_{5} \boldsymbol{E}_{\boldsymbol{x}, \boldsymbol{d o m}}^{\boldsymbol{j} \boldsymbol{I I}}$ exprime o número de emigrantes de data fixa, do período 1986/1991, cujo destino foram outras regiões do Brasil.

Resta estar atento a possíveis distorções nas estimativas de emigrantes internacionais, especialmente de regiões onde esse tipo de deslocamento populacional não apresenta grande expressão:

“... qualquer erro na população residente estimada de 1986 causará um erro de igual valor absoluto no saldo migratório do quinquênio 1981/1986 e um erro de igual proporção na população esperada de 1991. Esse último será transferido integralmente, em termos absolutos e com mesmo sinal, para a estimativa do número de emigrantes internacionais" (CARVALHO et al, 2001: 247).

Em suma, a precisão da estimativa do número de emigrantes internacionais dependerá, basicamente, do grau de cobertura dos Censos de 1980 e de 1991, da qualidade da informação censitária sobre migração de data fixa, da utilização de relações de sobrevivência representativas da área e do período em questão, e do rigor da estimativa da população residente no ano de 1986 (CARVALHO et al, 2001).

\section{3- VALADARES E IPATINGA: EMIGRANTES INTERNACIONAIS, SALDOS E TAXAS LÍQUIDAS DE MIGRAÇÃO}

Os procedimentos metodológicos tratados na seção anterior permitiram estimar o número de emigrantes internacionais, os saldos e as taxas líquidas de migração da microrregião de Valadares e da microrregião de Ipatinga.

Cadernos do Leste

Artigos Científicos

Belo Horizonte, Edição Especial, 2000 a 2008 
A respeito das informações que serviram de base para tais estimativas, cabe dizer que a distribuição, por sexo e grupo etário, da população brasileira e das duas microrregiões mineiras, em 1970, 1980 e 1991, foi retirada do censo, observando, neste caso, critério retroativo: as microrregiões foram reconstituídas em 1970 e em 1980, de acordo com os municípios que as integravam em 1991.

Com esses dados, foram obtidas as razões intercensitárias do Brasil, referentes aos períodos 1970/1980 e 1980/1990, bem como as razões de sobrevivência quinquenais, estimadas pelo método "Relação de Coortes" de Duchesne, para encontrar a população residente estimada da microrregião de Valadares e da microrregião de Ipatinga, em 1986.

Para dar conta das relações de sobrevivência masculina e feminina $\left(\mathrm{L}_{\mathrm{x}}\right)$, de cada uma das microrregiões, dois procedimentos foram adotados: i) o primeiro, compreendeu a utilização da técnica de mortalidade infanto-juvenil de $\operatorname{BRASS}^{7}(1974,1975)$ para encontrar o nível de mortalidade; e ii) o segundo consistiu em recurso à transformação logital, elaborado por BRASS (SHRYOCK \& SIEGEL, 1976; ORTEGA, 1987), para gerar as tabelas de sobrevivência de Valadares e de Ipatinga. As tabelas de sobrevivência de Minas Gerais, por sexo, desenvolvidas pelo Cedeplar, baseadas nos dados de óbitos do Sistema de Informação sobre Mortalidade - SIM - do Ministério da Saúde, óbitos esses corrigidos por fatores de correção de sub-registros determinados por meio das técnicas de Growth Balance e de PRESTON \& COALE (UNITED NATIONS, 1983), forneceram o padrão de mortalidade que permitiu o ajuste logital. As relações de sobrevivência $\left(\mathrm{L}_{\mathrm{x}}\right)$ do Brasil, relativas aos anos 70 e aos anos 80, foram retiradas de CARVALHO \& PINHEIRO (1986) e SAYWER et al (1999) respectivamente.

Esse conjunto de informações, associado com a razão intercensitária de sobrevivência da população observada no Brasil, na década de 70, leva ao conhecimento das relações intercensitárias de sobrevivência decenal, de sobrevivência do primeiro quinquênio e do

\footnotetext{
${ }^{7}$ A técnica de mortalidade infanto-juvenil de Brass “....consiste em procedimento que permite a conversão da proporção de crianças mortas, segundo a idade das mães na data do censo, em estimativas de probabilidade de morte $\left({ }_{x} \mathrm{q}_{0}\right)$ entre o nascimento e determinadas idades exatas, através de um conjunto de indicadores" (CARVALHO \& PINHEIRO, 1986).
}

Cadernos do Leste

Artigos Científicos

Belo Horizonte, Edição Especial, 2000 a 2008 
segundo quinquênio da população fechada da microrregião de Valadares e da microrregião de Ipatinga, nos anos 80 .

A configuração exibida pelos dados na TAB. 1 expressa a importância da emigração internacional na dinâmica populacional de Minas Gerais e da microrregião de Governador Valadares e da microrregião de Ipatinga. O número de pessoas residentes no exterior em 1991, com idade entre 10 e 64 anos $^{8,}$, que moravam, porém, no país, em Valadares e em Ipatinga, em 1986, seria da ordem de 176 400, de 12300 e de 10 800, respectivamente.

Tabela 1: Minas Gerias, Microrregião De Governador Valadares E Microrregião De Ipatinga - Emigrantes E Imigrantes Internacionais E Intranacionais, Por Sexo, Para O Período 1986/1991

\begin{tabular}{|c|c|c|c|c|c|c|c|c|c|}
\hline \multirow{2}{*}{ CATEGORIA } & \multicolumn{3}{|c|}{ MINAS GERAIS } & \multicolumn{3}{|c|}{ GOVERNADOR VALADARES } & \multicolumn{3}{|c|}{ IPATINGA } \\
\hline & Homens & Mulheres & Total & Homens & Mulheres & Total & Homens & Mulheres & Total \\
\hline Emigrantes internacionais & 109.607 & 66.814 & 176.421 & 7.131 & 5.205 & 12336 & 7.881 & 2.961 & 10.842 \\
\hline Imigrantes internacionais & 2.607 & 1.579 & 4.186 & 280 & 168 & 448 & 38 & 44 & 82 \\
\hline Emigrantes intranacionais & 206.635 & 206.737 & 413.372 & 16.436 & 16.571 & 33.007 & 11.358 & 12.016 & 23.374 \\
\hline Imigrantes intranacionais & 156.172 & 152.649 & 308.821 & 9.923 & 11.144 & 21.067 & 10.818 & 11.304 & 22.122 \\
\hline
\end{tabular}
NOTA: As estimativas referem-se às pessoas com idade entre 10 e 64 anos, em 1991.

Revelam os dados que a participação das microrregiões, na emigração internacional de Minas, foi significativa: ambas teriam contribuído com mais de 13\% desse fluxo no período 1986/1991, em face de uma população que representava, em 1991, pouco mais de 5\% da população estadual.

Se a distribuição, por sexo, dos emigrantes internacionais de Minas e de Valadares não manifesta grandes diferenças, não é o que ocorre em relação a Ipatinga: no fluxo mineiro e no valadarense, a contribuição dos homens teria girado ao redor de $62 \%$ e $58 \%$ respectivamente; em Ipatinga, os homens corresponderiam a proporção bem maior, quase $73 \%$. Cabe admitir

\footnotetext{
${ }^{8}$ Nas estimativas dos emigrantes internacionais, foram considerados os grupos etários compreendidos entre 10 e 64 anos, em 1991, por serem mais confiáveis.

Cadernos do Leste

Artigos Científicos

Belo Horizonte, Edição Especial, 2000 a 2008
} 
que o elevado percentual de emigrantes internacionais do sexo masculino em Ipatinga constitui indicativo do caráter mais recente das saídas internacionais ipatinguenses. Existem evidências de que a seletividade está, em certa medida, relacionada à evolução do movimento migratório: a migração é mais altamente seletiva por sexo e idade nas fases pioneiras, e menos seletiva nas fases posteriores (RENNER \& PATARRA, 1980).

Quanto às demais categorias de migrantes, cabe notar a participação bem menor dos imigrantes internacionais de Ipatinga (1,9\%), perante Valadares $(10,7 \%)$, no total de pessoas de outros países que entraram no estado. As perdas intranacionais de Minas, de Valadares e de Ipatinga, no período 1986/1991, gravitaram em torno de 413 mil, de 33 mil e de 23 mil, respectivamente. Os ganhos líquidos intranacionais, no quinquênio 1986/1991, foram de aproximadamente 309 mil, em Minas, 21 mil, na microrregião de Valadares, e 22 mil, na de Ipatinga ${ }^{9}$.

A contribuição da migração interna e da internacional ganha evidência na TAB. 2, por meio da decomposição do saldo migratório do quinquênio 1986/1991 em saldo migratório internacional e intranacional. Para as unidades espaciais em questão, todas as modalidades de saldos migratórios compareceram, nesse quinquênio, com sinal negativo. A perda líquida total de população (saldo migratório total) em Ipatinga foi quase duas vezes menor do que em Valadares. As trocas migratórias entre Ipatinga e outros países foram muito maiores (perda líquida de 11 mil pessoas) do que entre essa microrregião e o restante do Brasil (cerca de 1 400 pessoas). Não se pode dizer o mesmo em relação a Valadares, pois, nesse caso, houve equilíbrio entre os dois saldos negativos (algo próximo de 12 mil pessoas). Já, no estado, houve prevalência das perdas líquidas internacionais de população (172 mil pessoas).

Tabela 2: Minas Gerias, Microrregião De Governador Valadares E Microrregião De Ipatinga - Saldos Migratórios Total, Intranacional E Internacional, Por Sexo,

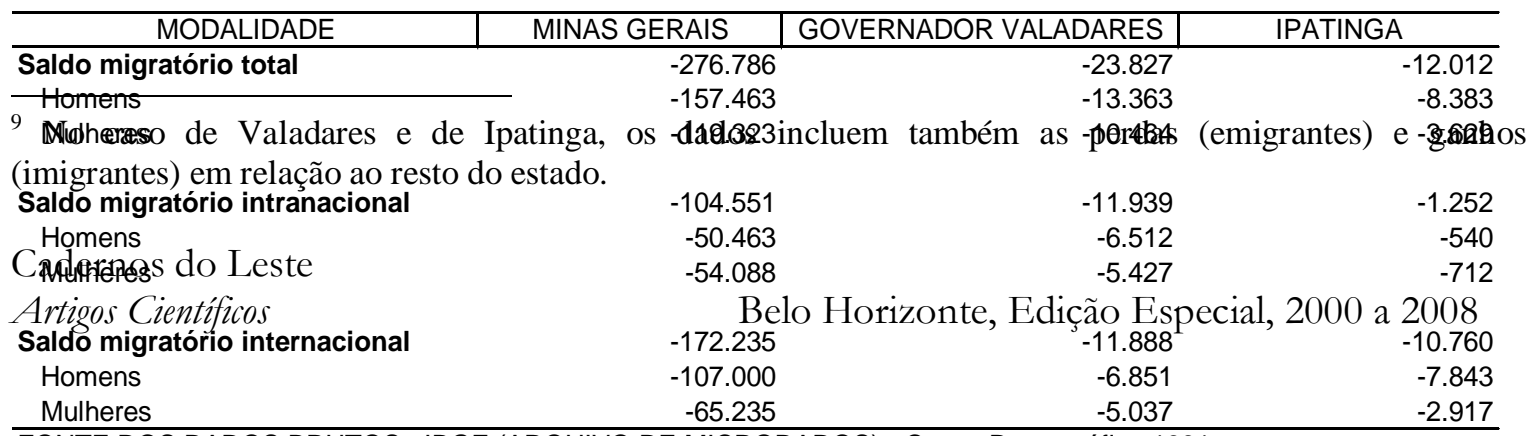

FONTE DOS DADOS BRUTOS: IBGE (ARQUIVO DE MICRODADOS) - Censo Demográfico 1991

NOTA: Saldos migratórios estimados para as pessoas com idade entre 10 e 64 anos, em 1991 


\section{Para O Período 1986/1991}

Os saldos migratórios estimados para o quinquênio 1981/1986 e para o decênio de 1981/1991 figuram na TAB. 3. Entre 1981 e 1986, as estimativas acusam perdas líquidas populacionais tanto para o estado quanto para as microrregiões: Minas teria perdido cerca de 266 mil pessoas, Valadares, 23 mil e Ipatinga, 11 mil.

A discriminação dos saldos migratórios, por sexo, não acusa grandes diferenças nas perdas líquidas de homens e mulheres, para Minas e Valadares - os números sugerem proporções equilibradas; a propósito de Ipatinga, a perda de homens (quase $70 \%$ ) foi bem maior do que a das mulheres.

Tabela 3: Minas Gerias, Microrregião De Governador Valadares E Microrregião De Ipatinga - Saldos Migratórios Quinquenais E Decenais, Por Sexo, Para O Período 1981/1991

\begin{tabular}{l|cc|c|c|c|c|c|c|c}
\hline \multirow{2}{*}{ PERÍODO } & \multicolumn{3}{|c|}{ MINAS GERAIS } & \multicolumn{2}{c}{ GOVERNADOR VALADARES } & \multicolumn{3}{c}{ IPATINGA } \\
\cline { 2 - 10 } & Homens & Mulheres & Total & Homens & Mulheres & Total & Homens & Mulheres & Total \\
\hline \multirow{2}{*}{$1981 / 1986$} & -148.187 & -117.346 & -265.533 & -12.690 & -10.131 & -22.821 & -7850 & -3385 & -11235 \\
$1986 / 1991$ & -157.463 & -119.323 & -276.786 & -13.363 & -10.464 & -23.827 & -8383 & -3629 & -12012 \\
$1981 / 1991$ & -302.391 & -244.160 & -546.551 & -26.351 & -21.566 & -47.917 & -16675 & -7788 & -24463 \\
\hline
\end{tabular}

FONTE DOS DADOS BRUTOS: IBGE (ARQUIVO DE MICRODADOS) - Censo Demográfico 1991

NOTA: Saldos migratórios estimados para as pessoas com idade entre 10 e 64 anos, em 1991

No decênio 1981/1991, as estimativas dão conta de que Minas teria tido perda líquida cerca de 302 mil homens e 244 mil mulheres, ao passo que, em Valadares, as perdas líquidas corresponderiam a 26 mil homens e 22 mil mulheres e, em Ipatinga, a 17 mil e 8 mil. Os respectivos quocientes das relações de cada um desses saldos com a população observada em 1991, taxa líquida de migração do decênio, revelam que, na microrregião de Governador Valadares, a população masculina deveria ser, em 1991, 14,2\% maior e a feminina 11\% maior; em Ipatinga, o número de homens, 8,2\% maior e o de mulheres, 3,7\% maior, na ausência de 
migrações nos anos 80. No estado, a população masculina e a feminina seriam, respectivamente, $3,9 \%$ e $3,1 \%$ maior.

\section{4- CONCLUSÃO}

A importância da migração internacional na dinâmica demográfica mineira, na valadarense e na ipatinguense, torna-se evidente pelo que ela representa no tocante às perdas líquidas ocorridas entre 1986 e 1991: no estado, a migração internacional respondeu por cerca de 62\% das perdas líquidas (172 mil, de 277 mil pessoas), em Valadares, por 50\% (12 mil, de 24mil pessoas), e, em Ipatinga, por 90\% (11mil, de 12 mil pessoas). Daí fica evidente o equilíbrio entre o saldo migratório intranacional e o internacional de Valadares e a prevalência do saldo migratório internacional em Ipatinga e Minas.

Se a diferença entre os emigrantes internacionais de Valadares e de Ipatinga não foi grande, o mesmo não pode ser dito da distribuição desses emigrantes por sexo: em Ipatinga, a participação relativa dos homens (quase 73\%) foi bem superior à das mulheres no fluxo internacional, o que indica o caráter mais recente das saídas internacionais dessa microrregião.

Tanto para o estado quanto para a microrregião de Valadares e de Ipatinga, as estimativas mostraram saldos migratórios negativos no quinquênio 1981/1986: as perdas líquidas de Minas, de Valadares e de Ipatinga foram, respectivamente, 266 mil, 23 mil e 11 mil pessoas. Verifica-se, portanto, que os saldos migratórios dos quinquênios 1981/1986 e 1986/1991 não revelam mudança de sinal nem alteração significativa no volume e na distribuição por sexo ao longo dos anos 80 .

Cadernos do Leste

Artigos Cientificos

Belo Horizonte, Edição Especial, 2000 a 2008 


\section{REFERÊNCIAS BIBLIOGRÁFICAS}

BRASS, Willian. Métodos para estimar la fecundidad y la mortalidad en pobraciones con datos limitados. Santiago de Chile: CELADE, 1974. 240p.

CARVALHO, José Alberto M. de. Migrações internas: mensuração direta e indireta. In: ENCONTRO NACIONAL DE ESTUDOS POPULACIONAIS, 2, 1980, Águas de São Pedro. Anais ... Belo Horizonte: ABEP, 1980. p. 533-577.

CARVALHO, José Alberto M. de. Estimativas indiretas e dados sobre migrações: uma avaliação conceitual e metodológica das informações censitárias recentes. Revista Brasileira de Estudos de População, Campinas, v.2, n.1, p.31-73, jan./jun. 1985.

CARVALHO, José Alberto M. de, PINHEIRO, Sílvia de Menezes Gama. Fecundidade e mortalidade no Brasil - 1970/1980. Belo Horizonte, UFMG/ CEDEPLAR, , 1986. 151p. (Relatório de pesquisa/CEDEPLAR).

CARVALHO, José Alberto M. de, MACHADO, Cláudio Caetano. Quesitos sobre migração no Censo Demográfico de 1991. Revista Brasileira de Estudos de População, Campinas, v.9, n.1, p.22-34, jan./jul.1992.

CARVALHO, José Alberto M. de. Crescimento populacional e estrutura demográfica no Brasil. Belo Horizonte: UFMG/CEDEPLAR, [199-]. 20p. (Trabalho apresentado no seminário sobre "Crescimento Populacional e Estrutura Demográfica", organizado pela Agência Brasileira de Cooperação/Ministério das Relações Exteriores - Rio de Janeiro, 27 e 28 de maio de 1993)

CARVALHO, José Alberto M. de. O saldo dos fluxos migratórios internacionais do Brasil na década de 80 : uma tentativa de estimação. Revista Brasileira de Estudos de População, Campinas, v.13, n.1, p.3-14, jan./jun. 1996.

CARVALHO, José Alberto M. de et al. Dados de migração de última etapa e data fixa do Censo Demográfico Brasileiro de 1991: uma análise preliminar de consistência. In: ENCONTRO NACIONAL DE ESTUDOS POPULACIONAIS, 11, 1998, Caxambu. Anais... Belo Horizonte: ABEP, 1998a. (disponível CD-ROM).

CARVAlHO, José Alberto M. de et al. Minas Gerais, uma nova região de atração populacional?. In: SEMINÁRIO SOBRE A ECONOMIA MINEIRA, 8, 1998, Diamantina. Anais... Belo Horizonte: UFMG/CEDEPLAR, 1998b. p.397-420.

Cadernos do Leste

Artigos Cientificos

Belo Horizonte, Edição Especial, 2000 a 2008 
CARVALHO, José Alberto M. de, RIGOTTI, José Irineu Rangel. Os dados censitários sobre migrações internas: algumas sugestões para a análise. Revista Brasileira de Estudos de População, Campinas, v.15, n.2, p.7-17, jul./dez. 1999.

CARVALHO, José Alberto M. de et al. Minas Gerais e a região de planejamento VIII - Rio Doce: emigrantes internacionais e saldos migratórios da década de 1980. In: SEMINÁRIO SOBRE A ECONOMIA MINEIRA, 9, 2000, Diamantina. Anais... Belo Horizonte: CEDEPLAR/UFMG, 2000a. v.2, p.843-884

CARVALHO, José Alberto M. de et al. Paraná: emigrantes internacionais de 1986/1991 e de 1991/1996 e saldos migratórios qüinqüenais entre 1981 e 1996. 2000b. (Trabalho apresentado no Encontro da Associação Nacional de Pós-Graduação e Pesquisa em Ciências Sociais, 12., Petrópolis, 2000).

CARVALHO, José Alberto M. de et al. Sinuosos caminhos para estimação do emigrantes internacionais de 1986/1991 e de 1991/1996 e saldos migratórios dos qüinqüênios entre 1981 e 1996 das Unidades da Federação Brasileira. In: ENCONTRO NACIONAL DE ESTUDOS POPULACIONAIS, 12., 2000, Caxambu. Brasil 500 anos: mudanças e continuidades. [Campinas]: ABEP, 2000c. (Disponível em CD-ROM)

CARVALHO, José Alberto M. de et al. Estimativas dos saldos migratórios internacionais e do número de emigrantes internacionais das grandes regiões do Brasil - 1986/1991 e 1991/1996. In: CASTRO, Mary Garcia. Migrações internacionais: contribuições para políticas. Brasília: CNPD, 2001. p.243-252

DUCHESNE, Louis (1989). Proyecciones de poblacíon por sexo y edad para áreas intermedias y menores: método "relación de cohortes". In: GRANADOS, Marai del Pilar. (Comp.) Métodos para proyecciones subnacionales de populacion. 1989. p. 71-126. RENNER, Cecília H., PATARRA, Neide L. Migrações. In: SANTOS, Jair L. Ferreira, LEVY, Maria Stella Ferreira, SZMRECSÃNYI, Tamás (Orgs). Dinâmica da população: teoria, métodos e técnicas de análise. São Paulo: T. A. Queiroz, 1980. p.21-85.

RIGOTTI, José Irineu Rangel. Técnicas de mensuração das migrações, a partir de dados censitários: aplicação aos casos de Minas Gerais e São Paulo. 1999. 142p. Tese (Doutorado em Demografia) - Centro de Desenvolvimento e Planejamento Regional, Universidade Federal de Minas Gerais, 1999.

SAWYER, Diana O. et al. Projeto temático I - Módulo: estimativas e projeções populacionais para o Brasil. In: CARVALHO, José Alberto Magno de (Coord.). Dinâmica demográfica, desenvolvimento regional e políticas públicas. Projeto PRONEX/CEDEPLAR, 1999. (Dados não publicados).

Cadernos do Leste

Artigos Cientificos

Belo Horizonte, Edição Especial, 2000 a 2008 
UNITED NATIONS. Manual VI: methods of measuring internal migration. New York: United Nations, 1970. 72p. (Population studies; 47) 